\title{
Case 2 - Adjuvant therapy in a patient with HER2-positive early breast carcinoma
}

\author{
Raúl Márquez Vázquez ${ }^{1}$, Antonio González Martín \\ Commentary: Javier Cortés
}

\begin{abstract}
Approximately $20 \%$ of breast cancer patients harbor tumors that overexpress human epidermal growth factor receptor 2 (HER2), which represents an adverse prognostic factor. The therapeutic landscape for this type of breast cancer has been transformed by the approval of anti-HER2 agents for adjuvant and neoadjuvant settings. Despite that, some of these patients will eventually relapse. Dual HER2 blockade has been successfully tried in early and advanced breast cancer. Here we report the case of an early HER2-positive breast cancer patient treated with trastuzumab, pertuzumab, and chemotherapy. The recently released final results of the much anticipated APHINITY trial confirm the outcome achieved in this patient.
\end{abstract}

Key words: adjuvant, breast cancer, HER2, pertuzumab

\section{Introduction}

Approximately $15-20 \%$ of breast cancers are human epidermal growth factor receptor 2 (HER2)-positive, which is associated with aggressive disease and poor prognosis [1]. Combining trastuzumab with adjuvant chemotherapy improves survival among patients with HER2-positive early breast cancer, reducing the risk of recurrence and death. However, up to one in four people $(25 \%)$ treated with trastuzumab and chemotherapy eventually relapse [2-4], and new approaches are needed. Dual blockage with trastuzumab and pertuzumab is an innovative strategy to enhance the clinical effectiveness of either agent administered singly. In randomized studies in the neoadjuvant setting, trastuzumab and chemotherapy-containing regimens have improved pathological complete response (pCR) at the time of surgery, which appears to correlate with improved disease outcomes. Thus, pCR may be able to serve as a surrogate marker of clinical benefit [5-7].

${ }^{1}$ Medical Oncology Department, MD Anderson Cancer Center, Madrid, Spain.

${ }^{2}$ Medical Oncology Department, Clínica Universidad de Navarra, Madrid, Spain.

Correspondence to:

Antonio González Martin

C/ Marquesado de Santa Marta 5, 28022 Madrid.

Phone: +34 913531920

E-mail: agonzalezma@unav.es

CANCER BREAKING NEWS 2017;5(3):36-40

DOI: $10.19156 / \mathrm{cbn} .2017 .0058$
Moreover, the combination of pertuzumab plus trastuzumab plus docetaxel, when used as first-line treatment for HER2-positive metastatic breast cancer, significantly prolonged progression-free survival compared with placebo plus trastuzumab plus docetaxel, and improved overall survival by 15.7 months [8].

Pertuzumab, a novel monoclonal antibody that functions as an anti-HER2 agent by targeting the extracellular dimerization domain of the HER2 receptor, was the first drug to receive, on September 30, 2013, accelerated approval by the US Food and Drug Administration for use in neoadjuvant settings in locally advanced, inflammatory, or early-stage breast cancer (either greater than 2 $\mathrm{cm}$ in diameter or node positive) HER2-positive breast cancer, based on the results of two clinical trials [9, 10]. In the NeoSphere trial, pertuzumab and trastuzumab plus docetaxel significantly improved pCR compared with those in patients given trastuzumab with docetaxel or pertuzumab with docetaxel (45.8\% vs $29 \%$ vs $24 \%$, respectively; $p=0.01$ ) [9]. In the Tryphaena trial, patients were randomized to one of three regimens: 6 cycles of docetaxel, carboplatin, trastuzumab, and pertuzumab (DCTP); 3 cycles of FEC (5-fluorouracil, epirubicin, and cyclophosphamide), FEC followed by 3 cycles of docetaxel plus trastuzumab/pertuzumab (FEC $\rightarrow$ DTP); or 3 cycles of FEC followed by 3 cycles of docetaxel, all concurrently with trastuzumab/pertuzumab (FEC-TP $\rightarrow$ DTP), resulting in a pCR (breast) rate of $61.6 \%, 57.3 \%$, and $66.2 \%$, respectively [10]. 
The recently released final results of the much anticipated APHINITY trial, which explored the benefit of dual HER2 blockade in the adjuvant setting, confirm the outcome achieved in this patient.

\section{Case description}

A 49-year-old female presented with a $1.8 \mathrm{~cm}$ lump in her upper external right breast. Swollen lymph nodes were also identified in the right axilla. The pathological diagnosis was grade 2 invasive lobular carcinoma (ILC) and a positive ipsilateral lymph node. The biological features were estrogen receptor (ER)-negative, progesterone receptor $(\mathrm{PgR})$ negative, HER2 2+ (FISH overexpressed) and Ki-67 index $39 \%$. There was no evidence of systemic disease through computed tomography (CT) scan thorax-abdomen-pelvis and bone scintigraphy. The patient was finally diagnosed with primary T1N1M0 breast cancer and underwent conservative surgery and lymph node dissection of the axilla on July 3rd, 2012. The final pathological report showed a multifocal grade 3 ILC, with a bigger $(2.5 \mathrm{~cm})$ lump along with lobular carcinoma in situ (LCIS). There were 2 out 19 tumor-affected lymph nodes. No extranodal component was found in the lymph nodes. The patient was enrolled in the APHINITY trial and received adjuvant chemotherapy with 4 cycles of AC (doxorubicin/cyclophosphamide 60/600 $\left.\mathrm{mg} / \mathrm{m}^{2}\right) \mathrm{q} 3 \mathrm{w}$ and then 12 cycles of weekly paclitaxel (80 $\mathrm{mg} / \mathrm{m}^{2}$ ) along with trastuzumab $6 \mathrm{mg} / \mathrm{kg}$ and pertuzumab $420 \mathrm{mg} /$ placebo q3w (trastuzumab $8 \mathrm{mg} / \mathrm{kg}$ and pertuzumab $840 \mathrm{mg}$ loading dose). Mild toxicity was observed: grade 1 peripheral neuropathy and grade 1 diarrhea. There was no decrease in heart left ventricular ejection fraction. After chemotherapy, she received radiotherapy in February/March 2013 and had completed 52 weeks of treatment with trastuzumab and pertuzumab-placebo at time of reporting. Since then, the patient has been followed up, with no evidence of local or systemic relapse 5 years after the surgery.

\section{Discussion}

Up to $25 \%$ of patients with HER2-positive and nodepositive operable breast cancer will have a relapse within 10 years, despite the use of adjuvant chemotherapy and 1 year of adjuvant trastuzumab. This is still far from optimal, and new options and strategies are needed for our patients.
In this regard, the APHINITY trial explored the benefits of dual HER2 blockade in the adjuvant setting [11]. The recently-released much anticipated final results of APHINITY confirm the outcome achieved in this patient.

APHINITY (Adjuvant Pertuzumab and Herceptin IN Initial TherapY in Breast Cancer) is a phase 3, international, randomized, double-blind, placebo-controlled, two-arm study evaluating the efficacy and safety of pertuzumab plus trastuzumab and chemotherapy compared with trastuzumab and chemotherapy, as an adjuvant therapy in 4,805 patients after undergoing surgery for HER2-positive cancer (NCT01358877/BO25126/BIG 4-11) [11]. Patients received 1 year of chemotherapy and trastuzumab, plus either pertuzumab or placebo; chemotherapy comprised a number of standard anthracycline/ taxane sequences or a non-anthracycline (TCH) regimen. Radiotherapy and/or endocrine therapy could be initiated at the end of adjuvant chemotherapy. The primary efficacy endpoint of APHINITY is invasive disease-free survival (iDFS), with an expected 3-year iDFS rate of $89.2 \%$ and $91.8 \%$ (hazard ratio [HR] 0.75) in the placebo and pertuzumab arm, respectively. After a median follow-up of 45.4 months, analysis showed a $19 \%$ relative risk reduction in the pertuzumab arm (94.1\% vs 93.2\%; stratified HR 0.81; $\mathrm{p}=0.045$ ) [11].

Approximately $62 \%$ of both groups were node positive. In a predefined subgroup analysis of patients by nodal status, there was a $23 \%$ reduction in relative risk among the patients treated with pertuzumab compared with placebo (92\% vs 90.2\%; unstratified HR 0.77; $\mathrm{p}=0.019$ ). Correspondingly, in the approximately $36 \%$ of patients who were hormone-receptor-negative, pertuzumab was associated with a relative risk reduction of $24 \%$ versus placebo (3-year iDFS 92.8\% vs 91.2\%; unstratified HR 0.76; $\mathrm{p}=0.085)$.

\section{Acknowledgments}

The Authors thank Ray Hill, an independent medical writer, who provided native English editing and journal styling on behalf of HPS.

\section{Conflicts of Interest}

The Authors declare there are no conflicts of interest in relation to this article. 


\section{References}

1. Ross JS, Slodkowska EA, Symmans WF et al. The HER2 receptor and breast cancer: ten years of targeted antiHER-2 therapy and personalized medicine. Oncologist 2009;14(4):320-68.

2. Piccart-Gebhart MJ, Procter M, Leyland-Jones B et al. Trastuzumab after adjuvant chemotherapy in HER2-positive breast cancer. N Engl J Med 2005;353(16):1659-72.

3. Romond EH, Perez EA, Bryant J et al. Trastuzumab plus adjuvant chemotherapy for operable HER2-positive breast cancer. N Engl J Med 2005;353:1673-84

4. Slamon D, Eiermann W, Robert N et al. Adjuvant trastuzumab in HER2-positive breast cancer. N Engl J Med 2011;365(14):1273-83.

5. Gianni L, Eiermann W, Semiglazov V et al. Neoadjuvant chemotherapy with trastuzumab followed by adjuvant trastuzumab versus neoadjuvant chemotherapy alone, in patients with HER2-positive locally advanced breast cancer (the NOAH trial): a randomised controlled superiority trial with a parallel HER2-negative cohort. Lancet 2010;375(9712):377-84.

6. von Minckwitz G, Untch M, Blohmer JU, et al. Definition and impact of pathologic complete response on prognosis after neoadjuvant chemotherapy in various intrinsic breast cancer subtypes. J Clin Oncol 2012;30(15):1796-804.

7. Cortazar P, Zhang L, Untch M et al. Pathological complete response and long-term clinical benefit in breast cancer: the CTNeoBC pooled analysis. Lancet 2014;384(9938):164-72.

8. Baselga J, Cortes J, Kim SB et al. Pertuzumab plus trastuzumab plus docetaxel for metastatic breast cancer. N Engl J Med 2012;366(2):109-19.

9. Gianni L, Pienkowski T, Im YH et al. Efficacy and safety of neoadjuvant pertuzumab and trastuzumab in women with locally advanced, inflammatory, or early HER2-positive breast cancer (NeoSphere): a randomised multicentre, open-label, phase 2 trial. Lancet Oncol 2012;13(1):25-32.

10. Schneeweiss A, Chia S, Hickish T et al. Pertuzumab plus trastuzumab in combination with standard neoadjuvant anthracycline-containing and anthracycline-free chemotherapy regimens in patients with HER2-positive early breast cancer: a randomized phase II cardiac safety study (TRYPHAENA). Ann Oncol 2013;24(9):2278-84.

11. von Minckwitz D, Proctor M, de Azambuja E et al. for the APHINITY Steering Committee and Investigators. Adjuvant pertuzumab and trastuzumab in early HER2-positive breast cancer. N Engl J Med 2017;377(2):122-31.

\section{Commentary}

Although the prognosis for both human epidermal growth factor receptor 2 (HER2)-positive early and metastatic breast cancer has changed dramatically with the introduction of anti-HER2 therapies, many patients still relapse and will ultimately die as a consequence of their disease [1-3]. In the adjuvant scenario, 1-year trastuzumab based-therapy is now considered the standard of care for the majority of patients, even with very small tumors, due to the aggressiveness of this disease. Although there have been several studies designed to improve 1-year trastuzumab-based therapy outcomes, statistically significant results were generally not observed. Longer duration of trastuzumab was explored in the HERA trial, which demonstrated that 2 years of trastuzumab was not better than 1 year [4]. Moreover, other trials have been designed to explore if lower cycles of trastuzumab could be non-inferior to the 1-year schedules. At the time being, none of the data support shorter courses, and 1 year of trastuzumab remains as the standard of care [5, 6].

Adding a second drug to adjuvant trastuzumab has already been explored in 3 studies. In the ALTTO trial different strategies including lapatinib were compared with 1 year of trastuzumab. Without entering into detail in the clinical trial design, the study was negative for the primary endpoint, so lapatinib was unable to demonstrate any statistical significant benefit [7]. The ExteNET study explored the role of neratinib, an irreversible tyrosine kinase small-molecule inhibitor against the HER kinase family, in the adjuvant setting [8]. In brief, this phase III trial evaluated the efficacy of neratinib for 12 months compared with placebo in patients who had previously completed their adjuvant trastuzumab-based treatment, remained disease free, and were considered at ongoing risk of recurrence. The primary endpoint was invasive disease-free survival (iDFS). The primary analysis demonstrated a significantly superior outcome for patients taking neratinib with a 2-year iDFS of $93.9 \%$ compared with placebo 91.6\%, translating to an HR of 0.67, $p=0.0091$. Efficacy in several predefined subgroups also demonstrated a significant benefit with neratinib therapy, including iDFS in patients with hormone-receptor-positive disease with an HR of 0.51, 
$p=0.0013$. Finally, the long-awaited APHINITY trial [9]: in this study, over 4,800 patients were randomized to receive 1 year of trastuzumab-based therapy plus either pertuzumab or placebo. The primary endpoint was iDFS. After a median follow-up of 45.6 months, patients who received pertuzumab-based therapy had better iDFS with an HR of 0.81, $p=0.045$. Of interest, the unstratified HR was 0.77 for the subgroup of patients with nodes involved, and it was 0.76 for hormonereceptor-negative tumors, always favoring the pertuzumab arm.

The clinical case presented above is of a 49-year-old female who underwent surgery and pathology revealed a multifocal grade 3 invasive lobular carcinoma, with a bigger lump sized at $2.5 \mathrm{~cm}$ and with 2 out 19 tumor-affected lymph nodes. Estrogen receptor (ER) and progesterone receptor were both negative and HER2 2+ (FISH overexpressed). The patient was included in the APHINITY trial and received chemotherapy plus trastuzumab and pertuzumab.

The results according to the primary endpoint were significant: APHINITY is a positive trial. However, will the new therapy become a standard-of-care? Is there clinical significance of the $0.9 \%$ absolute benefit? Are the results mature enough to be taken into account? Should pertuzumab be used only in high-risk node positive/hormone receptor negative HER2 breast cancer in the adjuvant setting? How can we define a high-risk HER2-positive breast cancer? Although many of these answers will become available over the next years, it is remarkable to highlight that the patient we discuss does have more opportunities to be free of disease because she received pertuzumab as well. And this is the key argument when considering adjuvant pertuzumab. Although this is true for the total population, it is more important for patients with hormone receptor negative tumors and for positive axillary nodes. Indeed, this patient had these two features.

However, in my opinion, these data are still immature in some way. If we look in detail into the updated data from the different trastuzumab-based pivotal trials [8, 10], we can observe that a significant number of patients still relapse after the first 3-4 years of therapy. In addition, a significant number of patients with positive lymph nodes were included at the end of the APHINITY trial, with shorter follow-up. This means that it is quite likely that an important number of events will be observed in the updated analysis, with expected greater benefit in the pertuzumab arm. Although costs should also be taken into account when making any treatment decision, I would like to leave this question open until having updated data that will help us to make decisions that are more adequate.

So, in conclusion, based on the APHINITY trial, pertuzumab should be considered for a group of patients with high-risk HER2-positive early breast cancer. Patients with tumors larger than 2 $\mathrm{cm}$ and hormone receptor-negative tumors and/or patients with axilla involvement clearly benefit from pertuzumab in combination with trastuzumab-based therapy and, in my opinion, this new strategy should be offered. 


\section{References}

1. Piccart-Gebhart MJ, Procter M, Leyland-Jones B et al. Trastuzumab after adjuvant chemotherapy in HER2-positive breast cancer. N Engl J Med 2005;353(16):1659-72.

2. Romond EH, Perez EA, Bryant J et al. Trastuzumab plus adjuvant chemotherapy for operable HER2-positive breast cancer. N Engl J Med 2005;353(16):1673-84.

3. Slamon D, Eiermann W, Robert $\mathrm{N}$ et al. Adjuvant trastuzumab in HER2-positive breast cancer. N Engl J Med 2011;365(14):1273-83.

4. Goldhirsch A, Gelber RD, Piccart-Gebhart MJ et al. 2 years versus 1 year of adjuvant trastuzumab for HER2-positive breast cancer (HERA): an open-label, randomised controlled trial. Lancet 2013;382(9897):1021-8.

5. Pivot X, Romieu G, Debled $M$ et al. 6 months versus 12 months of adjuvant trastuzumab for patients with HER2positive early breast cancer (PHARE): a randomised phase 3 trial. Lancet Oncol 2013;14(8):741-8.

6. Conte PF, Bisagni G, Frassoldati A et al. 9 weeks versus 1 year adjuvant trastuzumab in combination with chemotherapy: results of the phase III multicentric Italian ShortHER study. ASCO 2017.
7. Piccart-Gebhart M, Holmes E, Baselga J et al. Adjuvant lapatinib and trastuzumab for early human epidermal growth factor receptor 2-positive breast cancer: Results from the randomized phase III Adjuvant Lapatinib and/or Trastuzumab Treatment Optimization Trial. J Clin Oncol 2016;34(10):1034-42.

8. Chan A, Delaloge S, Holmes FA et al. Neratinib after trastuzumab-based adjuvant therapy in patients with HER2-positive breast cancer (ExteNET): a multicentre, randomised, double-blind, placebo-controlled, phase 3 trial. Lancet Oncol 2016;17:367-77.

9. von Minckwitz G, Procter M, de Azambuja E et al. Adjuvant pertuzumab and trastuzumab in early HER2-positive breast cancer. N Engl J Med 2017;377(2):122-31.

10. Slamon D, Eiermann W, Robert N et al. Abstract S5-04: Ten year follow-up of BCIRG-006 comparing doxorubicin plus cyclophosphamide followed by docetaxel (AC-T) with doxorubicin plus cyclophosphamide followed by docetaxel and trastuzumab (AC-TH) with docetaxel, carboplatin and trastuzumab (TCH) in HER2+ early breast cancer [Abstract]. Cancer Res 2016;76(4 Supplement):SABCS15-S5-04. 